\title{
Perfil metabólico de ovelhas gestantes recebendo fontes lipídicas com diferentes sítios de degradação
}

\author{
Luciana Melo Sousa ${ }^{1 *}$; Maria Júlia Pereira de Araújo²; Adriana Lima Silva ${ }^{3}$; Marco Túlio Santos Siqueira ${ }^{4}$; \\ Luciano Fernandes Sousa ${ }^{5}$; Gilberto de Lima Macedo Júnior ${ }^{6}$;
}

DOI: https://doi.org/10.35699/2447-6218.2020.19570

\begin{abstract}
Resumo
Objetivou-se avaliar o efeito da inclusão de fontes lipídicas com diferentes sítios de degradação sobre o perfil metabólico de ovelhas gestantes. O experimento foi realizado na Universidade Federal de Uberlândia, na Fazenda Experimental Capim Branco, durante os meses de agosto a novembro de 2015, tendo 90 dias de duração. Foram utilizadas 24 ovelhas gestantes com peso corporal médio de 50,8 $\pm 0,7 \mathrm{~kg}$ e idade aproximada de $12 \pm 1$ meses. Os animais foram divididos em três tratamentos: Controle, Nutrigordura ${ }^{\circledR}$ e Caroço de Algodão. $\mathrm{O}$ volumoso utilizado foi a silagem de milho, e a relação volumoso:concentrado foi de 60:40 (na matéria seca). As coletas de sangue para determinação dos metabólitos proteicos, energéticos e hepáticos foram feitas a cada 15 dias e as análises da concentração de betahidroxibutirato (BHB) foram feitas aos 120, 135 e 140 dias de gestação. O delineamento utilizado foi inteiramente casualizado com medidas repetidas ao tempo. Houve diferença estatística no perfil metabólico energético das ovelhas entre os tratamentos para lipoproteína de alta densidade (HDL) e betahidroxibutirato (BHB) no pré-parto. Para o colesterol e lipoproteína de baixa densidade (LDL) houve interação entre tratamento e período. Não houve diferenças significativas para os metabólitos hepáticos em função dos tratamentos, apenas para o período. Em relação ao metabolismo proteico, houve interação para a variável ácido úrico. A suplementação lipídica com diferentes perfis de degradação foi eficiente em manter o metabolismo energético, hepático e proteico dos animais alterando a concentração de alguns metabólitos no sangue durante os terços médio e final da gestação.
\end{abstract}

Palavras-chave: Gestação. Lipídeos. Metabolismo. Ovinos.

\section{Metabolic profile of pregnant sheeps fed fat sources with different degradation sites}

\author{
Abstract

\footnotetext{
${ }^{1}$ Universidade Estadual Paulista Júlio de Mesquita Filho. Campus de Jaboticabal. Jaboticabal, SP. Brasil.

https://orcid.org/0000-0003-1016-8248

2Universidade Federal de Uberlândia. Campus Umuarama. Uberlândia, MG. Brasil.

https://orcid.org/0000-0002-7730-5828

${ }^{3}$ Universidade Federal de Uberlândia. Campus Umuarama. Uberlândia, MG. Brasil.

https://orcid.org/0000-0002-9153-3317

${ }^{4}$ Universidade Federal de Uberlândia. Campus Umuarama. Uberlândia, MG. Brasil.

https://orcid.org/0000-0002-2098-8568

${ }^{5}$ Universidade Federal do Tocantins. Araguaína, TO. Brasil.

https://orcid.org/0000-0002-6072-9237

${ }^{6}$ Universidade Federal de Uberlândia. Campus Umuarama. Uberlândia, MG. Brasil.

https://orcid.org/0000-0001-5781-7917

*Autor correspondente: lumelosousa@gmail.com
}

It was aimed to evaluate the effect of including fat sources with different digestion sites on the metabolic profile of pregnant sheep. The experiment was conducted at the Federal University of Uberlândia, at the experimental farm Capim Branco, from August to November 2015, in a experimental period of 90 days. Twenty-four pregnant ewes with an average body weight of $50.8 \pm 0.7 \mathrm{~kg}$ and approximate $12 \pm 1$ months old were used. The animals were allocated into three treatments: Control, Nutrigordura ${ }^{\circledR}$ and Cottonseed. Corn silage was used as the roughage, and the

Recebido para publicação em 09 de março de 2020 Aceito para publicação em 05 de junho de 2020

e-ISSN: 2447-6218 / ISSN: 2447-6218. Atribuição CC BY. 
Sousa, L. M. et al.

roughage:concentrate ratio was 60:40 (dry matter). Blood samples were taken to determine protein, energetic and hepatic metabolites every 15 days and the analyzes of betahydroxybutyrate (BHB) concentration were performed at 120, 135 and 140 days of pregnancy. The experimental design was completely randomized with repeated measures in time. Statistical difference was shown in the metabolic energetic profile of the sheep between the treatments for high-density lipoprotein (HDL) and betahydroxybutyrate (BHB) in the prepartum. It was found an interaction between treatment and period for cholesterol and low-density lipoprotein (LDL). There were no significant differences for hepatic metabolites due to treatments, only for periods. Referring to protein metabolism, there was an interaction for the uric acid variable, where fat supplementation with different degradation profiles was efficient in maintaining the energetic, hepatic and protein metabolism, changing the blood concentration of some metabolites during the middle and final thirds of pregnancy.

Key-words: Pregnancy. Lipids. Metabolism. Sheep.

\section{Introdução}

Os óleos vegetais e grãos de oleaginosas são as fontes lipídicas mais utilizadas na dieta de ruminantes, devido, principalmente, a proibição de se utilizar gorduras de origem animal na alimentação de ruminantes no Brasil desde a publicação da Portaria GM/MS n. 216, de 11 de julho de 1997. Segundo Berchielli et al. (2011), quando a gordura representa mais de $10 \%$ da energia metabolizável, em muitas espécies, o consumo de matéria seca tende a declinar, provavelmente ocasionado pelos limites metabólicos de utilização da gordura, tanto para oxidação quanto para armazenamento nos tecidos.

Dessa forma, tem se buscado alternativas para que a inclusão de lipídeos ocorra sem efeitos deletérios sobre os microrganismos ruminais, além do aproveitamento de uma fonte intacta à fermentação ruminal. A saponificação de ácidos graxos via formação de sais de cálcio é uma alternativa frente à essa realidade, visto que se formam moléculas menos hidrolisáveis no ambiente ruminal, passando em parte intactas ao intestino delgado.

Segundo Macedo Júnior et al. (2014), a gestação é uma fase muito importante na vida produtiva da ovelha, e pode ser dividida em dois períodos bem definidos. Além disso, devemos considerar que possam existir mudanças na metabolizabilidade, bem como na digestibilidade dos nutrientes e utilização destes durante a gestação (Resende, 2011). Em virtude disso, vem sendo empregados à utilização de parâmetros bioquímicos como ferramenta para avaliar o status metabólico, e assim estabelecer, por meio de dosagens sanguíneas, o grau de adequação fisiológica dos animais às principais vias metabólicas relacionadas com energia e proteína (Silva et al., 2018).

Diante do exposto, o presente estudo tem como objetivo avaliar o efeito da inclusão de fontes lipídicas com diferentes sítios de degradação sobre o perfil metabólico de ovelhas gestantes.

\section{Material e métodos}

O experimento foi realizado na fazenda experimental Capim Branco da Universidade Federal de Uberlândia, no setor de caprinos e ovinos, durante os meses de agosto a novembro de 2015. O protocolo experimental deste trabalho foi aprovado pela Comissão de Ética na Utilização de Animais (CEUA) da UFU sob o número 096/15. Foram utilizadas vinte e quatro ovelhas mestiças Santa Inês, vacinadas (clostridioses, leptospirose, raiva e botulismo) e vermifugadas (Monepantel), com idade média acima de $12 \pm 1$ meses e peso corporal médio de $50,8 \pm 0,7 \mathrm{~kg}$, ao início do período experimental.

Antes do período experimental, as mesmas foram sincronizadas com uso de hormônio (prostaglandina em aplicação única de $0,5 \mathrm{~mL}$ via intramuscular) e cobertas por monta natural com macho reprodutor da raça Dorper. Após confirmação da prenhez (30 dias pós cobertura), através de ultrassonografia, as ovelhas ficaram em piquetes $\left(800 \mathrm{~m}^{2}\right)$ com pasto de capim Urochloa brizantha cv Marandu de junho a agosto (até 60 dias de gestação) recebendo suplemento proteico e energético na forma de proteinado e com disponibilidade de água e sombra artificial. Durante esse período, as médias da precipitação $(\mathrm{mm})$, umidade (\%) e temperaturas máxima e mínima $\left({ }^{\circ} \mathrm{C}\right)$ foram de $8,2,60,0,26,7$ e 16,8 , respectivamente.

As avaliações experimentais foram feitas a partir dos 60 dias de gestação, aproximadamente, até a parição ( \pm 147 dias de gestação). Os animais foram confinados em três baias de piso ripado, sendo oito animais em cada baia, com disponibilidade de sal mineral, cocho externo e bebedouro de tambor de plástico (limpos e repostos todos os dias). Passaram por procedimento padrão de pesagem, identificação, avaliação da condição corporal, vermifugação e posteriormente foram sorteados nos tratamentos conforme Tabela 2.

Foram utilizados três tratamentos: controle (isento de gordura suplementar), Nutrigordura ${ }^{\circledR}$ (gordura inerte de palma) e caroço de algodão inteiro com línter no concentrado. Como volumoso foi utilizado silagem de milho. $\mathrm{O}$ alimento foi fornecido duas vezes ao dia (8 horas e 16 horas).As rações foram balanceadas segundo o National Research Concil (NRC, 2007) para ovelhas em gestação. As mesmas eram compostas por silagem de milho, farelo de milho, farelo de soja, diferentes fontes de gordura e mistura mineral específico para espécie 
Perfil metabólico de ovelhas gestantes recebendo fontes lipídicas com diferentes sítios de degradação

ovina. A relação volumoso:concentrado foi de 60\%:40\%.

A composição dos concentrados está apresentada na Tabela 1.

Tabela 1 - Ingredientes e suas proporções nos concentrados experimentais (\%)

\begin{tabular}{lccc}
\hline Ingredientes & Controle & Nutrigordura $^{\circledR}$ & Caroço de Algodão \\
\hline Farelo de milho & 73,40 & 65,32 & 68,60 \\
Farelo de soja & 24,60 & 24,68 & 19,40 \\
Sal mineral & 1,00 & 4,00 & 2,00 \\
Ureia & 1,00 & 1,00 & 1,00 \\
Gordura protegida de & - & 5,00 & - \\
palma & - & - & 9,00 \\
Caroço de algodão inteiro & & & \\
\hline
\end{tabular}

Perfil de ácidos graxos do Nutrigordura ${ }^{\circledR}$ e Caroço de Algodão*

\begin{tabular}{lccc}
\hline Ácido graxos (\%) & & Nutrigordura $^{\circledR 1}$ & Caroço de Algodão \\
\hline Palmítico & $16: 0$ & 18,17 & 23,27 \\
Oleico & $18: 1$ & 27,98 & 15,50 \\
Linoleico & $18: 2$ & 38,27 & 55,50 \\
Linolênico & $18: 3$ & 3,04 & 2,10 \\
\hline Saturados & & 25,80 & 42,72 \\
Insaturados & & 74,20 & 57,28 \\
Poli-insaturados & & 41,30 & 10,95 \\
Monoinsaturados & 32,90 & 46,33 \\
\hline
\end{tabular}

*Fonte: FEA Unicamp e PESCE, 2008; ${ }^{1}$ Dados fornecidos pelo fabricante.

A oferta de ração foi de 3,5\% do peso corporal, de forma que sobrasse $10 \%$ do total fornecido, havendo ajuste do consumo pela mudança de peso vivo das ovelhas a cada 21 dias.

As coletas de sangue foram realizadas quinzenalmente (60, 75, 90, 105, 120 e 135 dias de gestação), estas ocorreram no período da manhã antes da primeira alimentação, por venopunção da jugular com auxílio de vacuntainner e tubos sem anticoagulante. Logo após, as amostras de cada animal foram centrifugadas, pipetadas e armazenadas em criotuboseppendorfs ${ }^{\circledR}$ para posterior análise laboratorial usando kit comercial da Lab Test ${ }^{\circledR}$. Os metabólitos energéticos determinados foram: colesterol (mg/dL), triglicerídeo (mg/dL), HDL (lipoproteína de alta densidade, $\mathrm{mg} / \mathrm{dL})$ e frutosamina $(\mathrm{mg} / \mathrm{dL})$. As lipoproteínas VLDL (lipoproteína de densidade muito baixa, $\mathrm{mg} / \mathrm{dL}$ ) e LDL (lipoproteína de baixa densidade) foram calculadas através da fórmula proposta por Friedewald et al. (1972): VLDL = (triglicerídeos/5) e $\mathrm{LDL}=$ (colesterol total $-\mathrm{HDL}-\mathrm{VLDL})$. Os metabólitos hepáticos foram: aspartato aminotransferase (U/L), gama glutamiltranferase (U/L) e fosfatase alcalina (U/L). E os metabólitos proteicos foram: albumina $(\mathrm{g} / \mathrm{dL})$, proteína total $(\mathrm{g} / \mathrm{dL})$, creatinina $(\mathrm{mg} / \mathrm{dL})$, ureia $(\mathrm{mg} / \mathrm{dL})$ e ácido úrico $(\mathrm{mg} / \mathrm{dL})$.

Foram feitas análises da concentração de betahidroxibutirato (BHB) no sangue aos 120, 135 e 140 dias de gestação. Esse exame mede a quantidade de cetonas no sangue. Para a análise foi utilizado o aparelho Optium $\mathrm{Xceed}^{\circledR}$. Para a coleta foi necessária uma pequena quantidade de sangue, coletado do animal por venopunção da jugular com auxílio de uma seringa e agulha 25x7. Foi retirado $0,5 \mathrm{~mL}$ de sangue, desprezou-se algumas gotas afim de evitar contaminação, e logo depois adicionou uma gota na fita para leitura e, em seguida, obteve-se o resultado da concentração de BHB.

O delineamento utilizado foi o inteiramente casualizado com medidas repetidas ao tempo. Cada tratamento com oito repetições (animais). Para a comparação dos tratamentos foi usado o teste de $\mathrm{T}$ a $5 \%$ de erro e dos períodos foi utilizado o estudo de regressão $(\mathrm{P}<0,05)$.

\section{Resultados e discussão}

Na tabela 2 podem ser observadas as médias dos metabólitos energéticos. Observa-se diferença estatística para HDL entre tratamentos e para o colesterol e 
Sousa, L. M. et al.

LDL houve interação entre período e tratamento. Para o

período gestacional, apenas as relações CT/HDL e LDL/ HDL não apresentaram diferença significativa.

Tabela 2 - Média dos metabólitos energéticos em função dos tratamentos e do período gestacional

\begin{tabular}{|c|c|c|c|c|c|c|c|}
\hline Tratamento & $\begin{array}{c}\mathrm{TG}(\mathrm{mg} / \\
\mathrm{dL})\end{array}$ & $\begin{array}{c}\text { FRUT } \\
(\mathrm{mmol} / \mathrm{dL})\end{array}$ & $\begin{array}{c}\text { HDL } \\
(\mathrm{mg} / \mathrm{dL})\end{array}$ & \multicolumn{2}{|c|}{ VLDL (mg/dL) } & CT/HDL & LDL/HDI \\
\hline Controle & 22,78 & 177,64 & $12,19 \mathrm{~B}$ & & 4,55 & 5,02 & 3,60 \\
\hline Nutrigordura ${ }^{\circledR}$ & 25,82 & 178,21 & $15,00 \mathrm{~A}$ & & 5,80 & 4,66 & 3,28 \\
\hline Caroço de Algodão & 23,57 & 171,8 & $14,23 \mathrm{AB}$ & & 4,71 & 4,40 & 3,01 \\
\hline $\begin{array}{l}\text { Período (dias de } \\
\text { gestação) }\end{array}$ & $\mathrm{TG}^{1}$ & FRUT $^{10}$ & $\mathrm{HDL}^{2}$ & & $\mathrm{VLDL}^{3}$ & $\mathrm{CT} / \mathrm{HDL}$ & LDL/HDL \\
\hline 60 & 30,55 & 141,11 & 10,00 & & 6,06 & 4,14 & 2,55 \\
\hline 75 & 17,70 & 176,54 & 14,37 & & 3,54 & 3,12 & 1,98 \\
\hline 90 & 21,29 & 187,33 & 14,91 & & 4,25 & 4,56 & 3,26 \\
\hline 105 & 31,82 & 175,47 & 13,78 & & 6,36 & 5,06 & 3,55 \\
\hline 120 & 26,40 & 205,90 & 16,20 & & 5,28 & 5,07 & 3,43 \\
\hline 135 & 23,71 & 198,28 & 18,57 & & 4,74 & 5,05 & 3,50 \\
\hline Parto & 16,81 & 168,31 & 11,75 & & 3,36 & 6,98 & 5,62 \\
\hline MG & 23,90 & 175,81 & 13,77 & & 4,77 & 4,70 & 3,30 \\
\hline $\mathrm{CV}$ & 20,56 & 10,62 & 32,56 & & 20,45 & 31,58 & 39,32 \\
\hline $\mathrm{VR}^{*}$ & $9-47$ & $172 \pm 2^{* *}$ & $7-42$ & & $1,6-9,8$ & - & - \\
\hline \multicolumn{8}{|c|}{ Colesterol $(\mathrm{MG}=57,22 \mathrm{mg} / \mathrm{dL} ; \mathrm{CV}=20,71 \% ; \mathrm{VR}=13-117 \mathrm{mg} / \mathrm{dL})$} \\
\hline Tratamento/Período & & 75 & 90 & 105 & 120 & 135 & Parto \\
\hline Controle $^{4}$ & & 44,25 & 58,37 & 58,62 & 68,75 & $73,50 \mathrm{AB}$ & 58,66 \\
\hline Nutrigordura ${ }^{\circledR 5}$ & & 43,00 & 71,42 & 70,42 & 81,33 & $104,50 \mathrm{~A}$ & 68,60 \\
\hline Caroço de Algodão $^{6}$ & & 42,75 & 57,44 & 60,62 & 71,66 & $64,66 \mathrm{~B}$ & 76,40 \\
\hline \multicolumn{8}{|c|}{ LDL $(\mathrm{MG}=40,61 \mathrm{mg} / \mathrm{dL} ; \mathrm{CV}=25,97 \% ; \mathrm{VR}=4,3-95,5 \mathrm{mg} / \mathrm{dL})$} \\
\hline Tratamento/Período & & 75 & 90 & 105 & 120 & 135 & Parto \\
\hline Controle $^{7}$ & & 29,90 & 40,93 & 42,30 & 48,45 & $69,20 \mathrm{AB}$ & 45,45 \\
\hline Nutrigordura $^{\circledR 8}$ & & 27,92 & 52,40 & 44,82 & 67,66 & $98,40 \mathrm{~A}$ & 52,48 \\
\hline Caroço de Algodão ${ }^{9}$ & & 24,70 & 38,00 & 43,57 & 48,80 & $60,60 \mathrm{~B}$ & 59,32 \\
\hline
\end{tabular}

*Varanis (2018); **Kaneko et al. (2008);CT: Colesterol; TG: Triglicerídeos; FRUT: Frutosamina; HDL: lipoproteína de alta densidade; VLDL: lipoproteína de densidade muito baixa; LDL: lipoproteína de baixa densidade; MG: média geral; CV: coeficiente de variação; VR: Valores de referência; ${ }^{1} \mathrm{y}=12,103934+0,315254 \mathrm{x}-0,001775 \mathrm{x}^{2} \mathrm{R}^{2}=16,37 \% ;{ }^{2} \mathrm{y}=-10,787751+0,476988 \mathrm{x}-0,002102 \mathrm{x}^{2} \mathrm{R}^{2}=56,35 \% ;{ }^{3} \mathrm{y}=2,262057+0,065837 \mathrm{x}$ $-0,000367 x^{2} R^{2}=16,48 \% ;{ }^{4} y=-70,580357+2,215377 x-0,008856 x^{2} R^{2}=93,00 \% ;{ }^{5} y=-100,075170+2,855911 x-0,011041 x^{2} R^{2}=76,07 \% ;{ }^{6}$ $\mathrm{y}=14,884127+0,417275 \mathrm{x} \mathrm{R}^{2}=88,95 \% ;{ }^{7} \mathrm{y}=-62,487202+1,701769 \mathrm{x}-0,006214 \mathrm{x}^{2} \mathrm{R}^{2}=77,80 \% ;{ }^{8} \mathrm{y}=-100,030119+2,461114 \mathrm{x}-0,008904 \mathrm{x}^{2}$ $\mathrm{R}^{2}=64,41 \% ;{ }^{9} \mathrm{y}=-7,372857+0,472524 \mathrm{x} \mathrm{R}^{2}=96,13 \% ;{ }^{10} \mathrm{Y}:-36,113820+4,058656 \mathrm{X}-0,017698 \mathrm{X}^{2}, \mathrm{R}^{2}=75,70 \%$

A média geral da HDL apresentou-se dentro da faixa de valores normais preconizados por Varanis (2018). Em relação aos tratamentos, apresentou maior valor

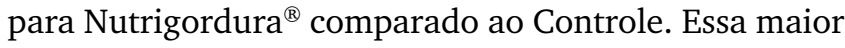
concentração pode estar relacionada com a presença de ácidos graxos insaturados, como o oleico e linoléico (Tabela 1), principalmente por se tratar de uma fonte de gordura inerte, ou seja, os lipídeos não sofreram biohidrogenação ruminal, chegando direto ao intestino delgado, sem alterar sua configuração no rúmen. Outro fator que pode ter aumentado o nível de HDL nesse tratamento foi a redução de amido na dieta (Tabela 1 ).O aumento da HDL traz benefícios ao animal, pois essa lipoproteína faz o transporte do colesterol para o fígado (Santos et al., 2015), 
Perfil metabólico de ovelhas gestantes recebendo fontes lipídicas com diferentes sítios de degradação

onde pode ser metabolizado e gerar energia ao animal. Em virtude disso, essa lipoproteína é chamada de "colesterol bom", uma vez que atua retirando o excesso de colesterol do organismo. No caso de consumo de alimentos ricos em gorduras, pode ocorrer, consequentemente, aumento dos níveis de HDL, como visto nesse estudo. Em relação ao período, nota-se aumento dos níveis com o avanço da gestação e queda no momento do parto. Essa menor concentração no momento do parto pode ser resultado de sua utilização pelo feto e, em menor extensão, pela utilização para a síntese de progesterona e de hormônios adrenais, uma vez que o momento do parto representa uma mudança hormonal muito importante para o animal, em que aumenta-se os níveis de estrógeno, GH e prolactina e diminui-se os níveis de progesterona. Santos et al., (2017) suplementando ovelhas gestantes no terço final com propilenoglicol verificou queda nos valores de HDL com a proximidade ao parto.

Para a variável colesterol houve interação entre tratamento e período. A média geral do colesterol apresentou-se dentro da faixa de valores normais segundo Varanis (2018). Houve aumento do colesterol em função do período em todos os tratamentos, semelhante ao que ocorreu com o HDL. Aos 135 dias o tratamento Nutrigordura ${ }^{\circledR}$ teve o maior valor de colesterol e depois uma queda grande no momento do parto. Já o tratamento com caroço de algodão apresentou menor valor aos 135 dias, porém aumentou ao parto, indicando que os animais conseguiram aumentar suas reservas de energia. De acordo com Raoofi et al., (2013), ocorre aumento gradual do colesterol em ovelhas no final da gestação, devido, principalmente, às concentrações de insulina, que atua diretamente no metabolismo do tecido adiposo durante o período gestacional, acarretando o aumento das concentrações de colesterol e das lipoproteínas carreadoras deste, como visto neste estudo a partir dos 105 dias de gestação (Tabela 2).O aumento de colesterol torna o animal energeticamente mais estável (com maior disponibilidade de energia) e tem forte relação com os níveis de betahidroxibutirato (BHB), que diminuíram com o avanço da gestação nesse estudo (Tabela 3 ).

Também houve interação entre tratamento e período para a LDL. A média geral encontra-se dentro do valor de referência (Varanis, 2018). Foi verificado maior valor dessa lipoproteína no tratamento com gordura inerte quando comparado ao caroço de algodão aos 135 dias de gestação, comportamento semelhante ao do colesterol. Em relação ao período, a LDL aumentou estatisticamente em todos os tratamentos e houve queda no momento do parto. O que pode ter provocado o aumento dessa variável à medida que se aproximava o parto é a formação de colesterol e excreção deste para o fígado. Ou seja, o animal mobilizou a reserva de triacilglicerol, levou para o fígado, o qual converteu parte em corpo cetônico e o restante em colesterol, levando ao aumento da LDL, do colesterol e, por consequência, do BHB (Tabela 3),visto que haveria mais colesterol no fígado precisando ser excretado para os demais tecidos.
Destaca-se que o aumento da LDL não é desejável ao animal uma vez que essa lipoproteína é responsável pelo transporte do colesterol e outros ácidos graxos do fígado para os demais tecidos do corpo (Santos et al., 2015), onde é acumulado e pode causar comprometimento de veias e artérias sanguíneas.

A média geral dos triglicerídeos apresentou-se dentro da faixa preconizada (Varanis, 2018). Em relação ao período nota-se queda dos níveis com o avanço da gestação, o que está relacionado com o aumento da LDL e também com o fato desses animais terem mobilizado reservas (sem causar danos aparentes, toxemia da gestação). Os triglicerídeos são moléculas que consistem em três cadeias longas de ácidos gordos esterificados para uma molécula de glicerol e têm por função basicamente estocar energia. Além disso, as menores concentrações de triglicérides observadas no final da gestação podem ser justificadas como resultante do aumento do aporte de triglicérides circulantes para a glândula mamária, sendo direcionado para a síntese de gordura do leite, da lipólise para obtenção de energia e da menor reserva de ácidos graxos livres disponíveis (Silva et al., 2013; Lima et al., 2016).

A lipoproteína VLDL encontrou-se dentro dos valores de referência (Varanis, 2018). E apresentou comportamento semelhante aos triglicerídeos. Isso ocorreu porque a VLDL é uma lipoproteína que transporta os triglicerídeos, dessa forma, já era esperado que esses metabólitos tivessem o mesmo padrão de resposta (Santos et al., 2015).

Em relação a frutosamina, essa se encontra $1 \%$ acima do recomendado por Kaneko et al. (2008), indicando que esses animais estavam energeticamente estáveis, o que reflete o aproveitamento energético dos animais. Em função do período é verificado aumento até os 135 dias de gestação e queda no momento do parto, comportamento semelhante ao colesterol e LDL. A frutosamina é uma cetoamina estável, formada quando a glicose reage não enzimaticamente com a proteína (albumina). Quando a concentração de proteína da dieta está dentro da normalidade, os índices de frutosamina estão relacionados aos níveis de glicose plasmática (Kaneko et al., 2008). No presente estudo, os metabólitos proteicos se encontram estáveis (Tabela 5), sugerindo que o que pode ter aumentado foi a glicose. Como dito anteriormente, o aumento gradual do colesterol se deve, principalmente, às concentrações de insulina, que atua diretamente no metabolismo do tecido adiposo durante o período gestacional. Esse aumento que ocorre nos níveis de insulina também pode ser estimulado pela glicose. Então, se a concentração de frutosamina aumentou ao longo do período, pode-se inferir que houve um aumento da glicose e com isso elevou também o colesterol e LDL, uma vez que a formação de colesterol necessita de glicose, bem como a elevação da glicose faz a LDL aumentar. 
Sousa, L. M. et al.

O betahidroxibutirato (BHB) e a glicose são metabólitos importantes no monitoramento do status energético em rebanho, especialmente durante a fase da gestação. O BHB é considerado como melhor indicador energético que a glicose, pois não possui controle homeostático tão estreito e é estável no soro, além de estar relacionado com a taxa de mobilização das reservas lipídicas durante o balanço energético negativo (BEN,
Feijó et al., 2014). Geralmente, é utilizado, durante as seis últimas semanas de gestação, como um indicador da presença de toxemia da gestação em estado inaparente (Jacondino et al., 2019). Foi observada diferença estatística no nível de BHB entre os tratamentos. Em relação ao período não houve diferença (Tabela 3). A média geral do BHB encontra-se dentro dos valores de referência (Taghipour et al., 2010).

Tabela 3 - Média do betahidroxibutirato em função dos tratamentos e do período gestacional

\begin{tabular}{|c|c|c|c|c|c|c|c|c|}
\hline \multicolumn{3}{|c|}{ Tratamento } & \multicolumn{3}{|c|}{ Período (dias de gestação) } & \multirow[b]{2}{*}{ MG } & \multirow[b]{2}{*}{$\mathrm{CV}$} & \multirow[b]{2}{*}{$\mathrm{VR}^{*}$} \\
\hline Controle & Nutrigordura $^{\circledR}$ & $\begin{array}{c}\text { Caroço de } \\
\text { Algodão }\end{array}$ & 120 & 135 & 140 & & & \\
\hline $0,58 \mathrm{~A}$ & $0,45 A$ & $0,16 \mathrm{~B}$ & 0,38 & 0,50 & 0,28 & 0,4 & 19,45 & $0,1-0,7$ \\
\hline
\end{tabular}

*Taghipour et al. (2010); MG: média geral; CV: coeficiente de variação; VR: valores de referência;

O tratamento com caroço de algodão apresentou menor valor de BHB comparado aos demais, que pode ser justificado pela velocidade de digestão da fonte lipídica. Esse resultado demonstra que esses animais mobilizaram menos gordura corporal, indicando que estavam mais energeticamente estáveis, semelhante aos resultados de frutosamina, colesterol e LDL (Tabela 2), já discutidos. Em relação ao período não houve diferença estatística, porém destaca-se o aumento da concentração aos 135 dias de gestação, comportamento semelhante ao que ocorre com a frutosamina, o colesterol e LDL (Tabela 2) no mesmo período. Essa ausência de diferença no período pode ser explicada pelos níveis de frutosamina, que aumentou até os 135 dias de gestação, deduzindo-se que a insulina possa ter aumentado. A toxemina da gestação é desencadeada pela queda na glicemia (Santos et al., 2011), o que reduz a insulina. Sendo assim, como foi observado, o colesterol aumentou até os 135 dias de gestação, indicando que a insulina manteve-se alta, não causando toxemia. É observado, também, que todos os tratamentos e períodos apresentaram valores abaixo de $0,7 \mathrm{mmol} / \mathrm{L}$, inferindo que esses animais tiveram baixa mobilização de reservas corporais ao final da gestação, devido, principalmente, às dietas utilizadas.

Em relação às enzimas hepáticas, não houve diferença entre os tratamentos para nenhuma das variáveis. Já, para o período gestacional houve, com exceção da aspartato aminotransferase (AST, Tabela 4). Foram observadas equações quadráticas para os metabólitos estudados em função do período, com exceção da AST. As equações quadráticas nos mostram que os animais diminuíram o nível dessas enzimas próximo ao parto (semelhante ao metabolismo energético, Tabela 2), devido à redução do consumo e como consequência houve redução na metabolização hepática. A aspartato aminotrasferase (AST) é uma enzima citoplasmática e mitocondrial, presente em vários tecidos como fígado, músculos esquelético e cardíaco. Essa enzima quando identificada acima das concentrações consideradas normais, indica que o animal pode desenvolver lesão hepato-celular secundária, oriundo da excessiva mobilização lipídica (Santos et al., 2015). No entanto, no presente estudo, suas concentrações estiveram dentro do intervalo considerado normal para a espécie (Varanis, 2018), o que indica que esses animais não desenvolveram lesão hepática.

A gama glutamiltransferase (GGT) é uma enzima usada como parâmetro bioquímico para indicar alterações hepáticas e biliares. Em todos os tratamentos e em relação ao período essa enzima se encontra dentro dos valores de referência descritos por Varanis (2018), indicando que o fígado desses animais encontrava-se em bom funcionamento e não apresentava possíveis alterações hepáticas. Em relação ao período gestacional, essa enzima aumentou suas atividades com o avanço da gestação e queda no momento do parto, semelhante ao colesterol, LDL e frutosamina, indicando aumento da atividade hepática nessa fase, justificado pelo aumento da demanda energética ao final da gestação, confirmando os achados de triglicerídeos e VLDL.

A fosfatase alcalina é uma enzima sintetizada em vários tecidos, sendo as maiores concentrações no intestino, rins, ossos e fígado (Kaneko et al., 2008). Aumento nos níveis de fosfatase alcalina no plasma sanguíneo é consequência de lesões nos tecidos hepáticos. No presente estudo, as concentrações de fosfatase alcalina encontram-se dentro dos valores de referência preconizados por Varanis (2018). As enzimas AST, GGT e fosfatase alcalina apresentaram-se dentro dos valores considerados normais para a espécie, indicando que esses animais não desenvolveram lesão hepática.

Na tabela 5 podem ser observadas as médias dos metabólitos proteicos. Não se observa diferença estatística em nenhuma das variáveis para tratamento. Em função do período gestacional houve diferença significativa para creatinina, ureia e ácido úrico.

Na avaliação da proteína total e albumina não foram observadas diferenças significativas entre os tratamentos e entre os períodos (Tabela 5), estando à média 
Perfil metabólico de ovelhas gestantes recebendo fontes lipídicas com diferentes sítios de degradação

geral dentro da normalidade (Varanis, 2018). A proteína sérica total e suas frações são consideradas como o indicador mais sensível para determinar o estado nutricional proteico, de modo que valores persistentemente baixos sugerem inadequado consumo proteico. Como visto, pode-se inferir que o consumo de proteína nesse estudo foi adequado, o que reforça o resultado de aumento na frutosamina ser pela elevação da glicose.

Tabela 4 - Média dos metabólitos hepáticos em função dos tratamentos e do período gestacional

\begin{tabular}{lccc}
\hline Tratamento & AST (U/L) & GGT (U/L) & Fosfatase alcalina (U/L) \\
\hline Controle & 111,27 & 62,68 & 136,35 \\
Nutrigordura $^{\circledR}$ & 104,39 & 68,52 & 153,65 \\
Caroço de Algodão $^{\circledR}$ & 117,35 & 71,08 & 142,30 \\
\hline Período (dias de gestação) & AST & GGT $^{1}$ & Fosfatase Alcalina $^{2}$ \\
\hline 60 & 90,94 & 50,11 & 100,05 \\
75 & 106,62 & 63,16 & 158,16 \\
90 & 122,70 & 61,62 & 151,12 \\
105 & 125,35 & 67,18 & 165,39 \\
120 & 108,20 & 88,60 & 158,10 \\
135 & 102,67 & 93,03 & 134,71 \\
Parto & 109,01 & 77,69 & 124,43 \\
\hline MG & 111,22 & 67,39 & 143,79 \\
CV & 36,96 & 24,62 & 31,66 \\
\hline VR* & $45-251$ & $28-104$ & $33-331$ \\
\hline
\end{tabular}

*Varanis (2018); AST: aspartato aminotransferase; GGT: gama glutamiltransferase; MG: média geral; CV: coeficiente de variação; VR: valores de referência; ${ }^{1} \mathrm{Y}=29,264802+0,403490 \mathrm{xR}^{2}=71,35 \% ;{ }^{2} \mathrm{Y}=-116,649119+5,265474 \mathrm{x}-0,024697 \mathrm{x}^{2} \mathrm{R}^{2}=80,49 \%$

No que diz respeito à ureia, não houve diferença estatística entre os tratamentos, contudo verifica-se aumento em função do período gestacional e redução próximo ao parto. Esse aumento se deve, principalmente, pelo fato de os animais estarem a pasto anteriormente e depois receberem concentrados com ureia em sua composição. A ureia responde mais rapidamente em relação à albumina a mudanças no aporte proteico da dieta para caracterização de deficiência proteica e sua concentração no soro sanguíneo reflete diretamente a quantidade de proteína ingerida (Silva et al., 2013). A ureia sanguínea reflete diretamente a amônia gerada no rúmen pela degradação dos compostos nitrogenados e que não foi convertida em proteína microbiana. Essa amônia excedente atravessa a parede ruminal, indo diretamente para ser transformada em ureia no fígado com alto gasto energético. Essa ureia pode retornar ao rúmen via saliva ou difusão na parede ruminal e ser eliminada na urina ou no leite, em caso de animais em lactação (Guimarães Júnior et al., 2016). Além disso, pode refletir na incapacidade de a microbiota fermentar as fontes de carboidratos em função dos efeitos deletérios do lipídeo. Ocasionando assim aumento da ureia ao longo do experimento. Contudo, a média geral da ureia encontra-se dentro dos valores de referência (Varanis, 2018). A redução da ureia próxima ao parto está relacionada com o fato de os animais diminuírem consumo de matéria seca devido, principalmente, à compressão ruminal.

Não foram observadas diferenças estatísticas em função do tratamento para a variável creatinina, contudo, nota-se diferença significativa em função do período gestacional. Os valores de creatinina estão dentro dos valores de referência descritos por Varanis (2018). A creatinina é um metabólito gerado a partir da quebra da creatina fosfato que é uma proteína muscular e sua função é atuar no funcionamento dos músculos. Além disso, quando os rins não estão funcionando bem os níveis de creatinina no sangue se elevam, então esse metabólico reflete insuficiência renal (Santos et al., 2011). A redução da creatinina ao longo do período relaciona-se com os achados para AST, GGT e fosfatase alcalina, confirmando que esses animais não apresentaram alterações renais e hepáticas durante o estudo. Ao dosar o metabólito de ovelhas Santa Inês no período final de gestação, Araújo (2009) observou o valor médio de $1,15 \mathrm{mg} / \mathrm{dL}$ e Nascimento et al. (2015), média de 0,95 mg/dL.

Em relação ao ácido úrico, houve interação entre os tratamentos e o período de gestação. O mesmo se encontra dentro dos valores de referência relatados por Varanis (2018). É observado aumento desse metabólito ao longo da gestação e queda no momento do parto em 
Sousa, L. M. et al.

todos os tratamentos. Sendo assim, pode-se inferir que à medida que aumentou o ácido úrico em todos os tratamentos ao longo do período, os animais aumentaram a formação de proteína microbiana, uma vez que $80 \%$ do ácido úrico é proteína microbiana (Paula, 2015), e consequentemente ao chegar no intestino delgado liberou aminoácidos e ácidos graxos, o que reforça a explicação da diminuição do BHB (Tabela 3). A queda do ácido úrico no momento do parto está associada à diminuição da capacidade de consumo desses animais próximo ao parto, originadas do rápido crescimento do útero gravídico e consequente limitação do consumo por falta de espaço, uma vez que esses metabólitos respondem rápido ao efeito da ração. O mesmo é observado para as variáveis creatinina e ureia.

Tabela 5 - Média dos metabólitos proteicos em função do tratamento e do período gestacional

\begin{tabular}{|c|c|c|c|c|c|c|c|}
\hline \multicolumn{2}{|l|}{ Tratamento } & $\underset{d \mathrm{dL})}{\text { Albumina }(\mathrm{g} /}$ & \multicolumn{2}{|c|}{ Creatinina $(\mathrm{mg} / \mathrm{dL})$} & $\begin{array}{l}\text { Ureia (mg/ } \\
\text { dL) }\end{array}$ & \multicolumn{2}{|c|}{$\begin{array}{l}\text { Proteína total }(\mathrm{g} / \\
\mathrm{dL})\end{array}$} \\
\hline Controle & & 3,46 & \multicolumn{2}{|c|}{0,48} & 35,2 & \multicolumn{2}{|c|}{5,34} \\
\hline Nutrigordura $^{\circledR}$ & & 3,58 & \multicolumn{2}{|c|}{0,52} & 29,05 & \multicolumn{2}{|c|}{5,29} \\
\hline Caroço de Algodão & & 3,60 & \multicolumn{2}{|c|}{0,56} & 31,47 & \multicolumn{2}{|c|}{5,57} \\
\hline Período (dias de gest & & Albumina & \multicolumn{2}{|c|}{ Creatinina $^{1}$} & Ureia $^{2}$ & \multicolumn{2}{|c|}{ Proteína total } \\
\hline 60 & & 3,22 & \multicolumn{2}{|c|}{0,83} & 9,33 & \multicolumn{2}{|c|}{5,44} \\
\hline 75 & & 3,46 & \multicolumn{2}{|c|}{0,49} & 37,87 & \multicolumn{2}{|c|}{4,96} \\
\hline 90 & & 3,76 & \multicolumn{2}{|c|}{0,37} & 39,54 & \multicolumn{2}{|c|}{5,50} \\
\hline 105 & & 3,60 & \multicolumn{2}{|c|}{0,43} & 40,13 & \multicolumn{2}{|c|}{5,72} \\
\hline 120 & & 3,21 & \multicolumn{2}{|c|}{0,56} & 30,90 & \multicolumn{2}{|c|}{5,34} \\
\hline 135 & & 3,60 & \multicolumn{2}{|c|}{0,54} & 30,71 & \multicolumn{2}{|c|}{5,26} \\
\hline Parto & & 3,81 & \multicolumn{2}{|c|}{0,54} & 26,96 & \multicolumn{2}{|c|}{5,53} \\
\hline MG & & 3,54 & \multicolumn{2}{|c|}{0,52} & 32,00 & \multicolumn{2}{|c|}{5,40} \\
\hline $\mathrm{CV}$ & & 12,01 & \multicolumn{2}{|c|}{30,79} & 27,16 & \multicolumn{2}{|c|}{10,07} \\
\hline $\mathrm{VR}^{*}$ & & $1,56-5,01$ & \multicolumn{2}{|c|}{$0,2-1,5$} & $7-55,8$ & \multicolumn{2}{|c|}{$2,26-7,18$} \\
\hline \multicolumn{8}{|c|}{ Ácido úrico $\left(\left(\mathrm{MG}=0,32 \mathrm{mg} / \mathrm{dL} ; \mathrm{CV}=35,89 \% ; \mathrm{VR}^{*}=0,1-0,9 \mathrm{mg} / \mathrm{dL}\right)\right.$} \\
\hline Tratamento/Período & 60 & 75 & 90 & 105 & 120 & 135 & Parto \\
\hline Controle & 0,21 & 0,18 & 0,32 & 0,57 & 0,37 & 0,4 & 0,24 \\
\hline Nutrigordura $^{\circledR}$ & 0,25 & 0,17 & 0,25 & 0,62 & 0,43 & 0,65 & 0,12 \\
\hline Caroço de Algodão & 0,30 & 0,17 & 0,32 & 0,58 & 0,56 & 0,33 & 0,08 \\
\hline
\end{tabular}

*Varanis (2018); MG: média geral; CV:coeficiente de variação; VR: Valores de referência; ${ }^{1} \mathrm{y}=1,952361-0,027576 \mathrm{x}+0,000125 \mathrm{x}^{2} \mathrm{R}^{2}=61,32 \%$; ${ }^{2} \mathrm{y}=-78,671180+2,186141 \mathrm{x}-0,010071 \mathrm{x}^{2} \mathrm{R}^{2}=67,24 \% ;{ }^{3} \mathrm{y}=-0,935417+0,024735 \mathrm{x}-0,000112 \mathrm{x}^{2} \mathrm{R}^{2}=59,05 \% ;{ }^{4} \mathrm{y}=-1,290459+0,032150 \mathrm{x}-$ $0,000145 x^{2} R^{2}=39,28 \% ;{ }^{5} y=-1,307103+0,034394 x-0,000165 x^{2} R^{2}=55,39 \%$;

Os metabólitos estudados apresentaram-se dentro dos valores de referência ao longo do período e em função do tratamento. Destaca-se o aumento do colesterol total e HDL no tratamento com gordura inerte, justificado pelo perfil de ácidos graxos da dieta, que proporcionou elevação das respectivas frações relativas ao metabolismo de lipídeos, transportadas no sangue (Freitas Júnior et al., 2010). E também à queda de triglicerídeos e VLDL ao final da gestação, desencadeado pela mobilização de reservas. A alta concentração de ácidos graxos livres, colesterol e triglicerídeos no sangue é resultante da mobilização de gordura corporal, o que permite sugerir esses como ferramentas de manejo nutricional, no período final de gestação, para auxiliar a identificação de animais potencialmente sujeitos a desordens metabólicas (Silva et al., 2018)

\section{Conclusão}

Baseado no exposto, conclui-se que a suplementação lipídica com diferentes perfis de degradação foi eficiente em manter o metabolismo energético, hepático e proteico dos animais alterando a concentração de alguns metabólitos no sangue durante o terço médio e final da gestação. 
Perfil metabólico de ovelhas gestantes recebendo fontes lipídicas com diferentes sítios de degradação

Aprovação do Comitê de Ética

O projeto foi aprovado pela Comissão de Ética na Utilização de Animais (CEUA) da UFU sob o número 096/15.

\section{Referências}

Araújo, C. A. S. C. 2009. Estudo comparativo do perfil metabólico e hormonal de ovelhas com gestação única, gemelar e não gestantes alimentadas com dieta de alta densidade energética. São Paulo: Universidade de São Paulo, 212f. Dissertação Mestrado. Disponível em: https://teses.usp.br/teses/disponiveis/10/10136/tde-21092010162531/fr.php.

Berchielli, T. T.; Pires, A. V.; Oliveira, S. G. 2011. Nutrição de Ruminantes. Funep, Jaboticabal, SP, BR.

Feijó, J. O.; Perazzoli, D.; Silva, L. G. C.; Aragão, R. B.; Martins, C. F.; Pereira, R. A.; Ferreira, M. B.; Pino, F. A. B. D.; Rabassa, V. R.; Correa, M. N. 2014. Avaliação de parâmetros bioquímicos clínicos de ovelhas do grupo genético pantaneiro gestantes e não gestantes. Brazilian Journal of Veterinary Research and Animal Science, 51: 111-117. Doi: 10.11606/issn.1678-4456.v51i2p111-117.

Freitas Júnior, J. E.; Rennó, F. P.; Prada e Silva, L. F.; Gandra, J. R.; Maturana Filho, M.; Foditsch, C.; Venturelli, B. C. 2010. Parâmetros sanguíneos de vacas leiteiras suplementadas com diferentes fontes de gordura. Ciência Rural, 40: 950-956. Doi: 10.1590/S010384782010005000039 .

Friedewald, W. T,; Levv, R. I.; Fredrickson, D. S. 1972. Estimation of the concentration of low-density lipoprotein in plasma, without use of the preparative ultracentrifuge. Clin Chem, 18: 499-502. Doi: 10.1136/jcp.s1-5.1.26.

Guimarães Júnior, R.; Pereira, L. G.; Tomich, T. R.; Machado, F. S.; Gonçalves, L. C. 2016. Informações gerais da ureia. Cadernos Técnicos de Veterinária e Zootecnia, 80: 9-25. Doi: 10.13140/RG.2.2.35545.52322.

Jacondino, L. R.; Gonçalves, A. S.; Riet Correa, B.; Oberst, E. R.; Silva, M. K.; Birgel, D. B.; Birgel Júnior, E. H.; Raimondo, R. F. S. 2019. Comparação de métodos para avaliação de beta-hidroxibutirato em ovelhas. Arquivo Brasileira de Medicina Veterinária e Zootecnia, 71: 857-862. Doi: 10.1590/1678-4162-10267.

Kaneko J. J.; Harvey J. W.; Bruss M. L. 2008. Clinical Biochemistry of Domestic Animals. 6. ed. Academic Press, San Diego, USA.

Lima, I. S. S.; Garcez, B. S.; Alves, A. A.; Aquino, F. C.; Borges, L. S.; Carvalho, W. F. 2016. Gordura protegida e perfil de ácidos graxos do leite de cabra: Uma revisão. Revista Brasileira de Higiene e Sanidade Animal, 10: 830-840. Doi: 10.5935/1981-2965.20160068.

Macedo Júnior, G. L.; Borges, I.; Cavalcanti, L. F. L.; Sousa, F. A.; Ferreira, M. I. C. 2014. Consumo, digestibilidade aparente e glicemia de borregas gestantes e submetidas a dois manejos nutricionais. Veterinária Notícias, 20: 57-70. Doi: 10.14393/VTv20n2a2014.24619.

Nascimento, P. M.; Morgado, A. A.; Nunes, G. R.; Nikolaus, J. P.; Weigel, R. A.; Lima, A. S.; Storillo, V. M.; Mori, C. S.; Sucupira, M. C. A. 2015. Metabolismo oxidativo e perfil bioquímico de ovelhas santa Inês no período periparto: efeito da suplementação parenteral com vitamina E. Semina: Ciências Agrárias, 36: 1397-1408. Doi: 10.5433/1679-0359.2015v36n3p1397.
National Research Council - NRC. 2007. Nutrient Requeriments of Small Ruminants. 1. ed. Washington, DC, USA.

Paula, C. G. 2015. Suplementação com melaço de soja na dieta de ovinos: parâmetros sanguíneos, consumo, digestibilidade e comportamento ingestivo. Uberlândia: Universidade Federal de Uberlândia, 61f. Dissertação Mestrado. Disponível em: https://repositorio.ufu.br/ handle/123456789/13154.

Raoofi, A.; Jafarian, M.; Safi, S.; Vatankhah, M. 2013. Fluctuations in energy-related metabolites during the peri-parturition period in Lori Bakhtiari ewes. Smal Ruminant Res., 109: 64-68. Doi: 10.1016/j. smallrumres.2012.06.012.

Resende, K. T.; Teixeira, I. A. M. A.; Fernandes, M. H. M. R. 2011. Metabolismo de energia. P. In: Berchielli, T. T.; Pires, A. V.; Oliveira, S. G., eds. Nutrição de Ruminantes. Funep, Jaboticabal, SP, BR.

Santos, R. P.; Macedo Junior, G. L.; Silva, S. P.; Sousa, L. F.; Andrade, M. E. B. 2017. A suplementação com propilenoglicol melhora o metabolismo energético em ovelhas gestantes. Revista Brasileira de Ciências Agrárias, 12: 561-566. Doi: 10.5039/agraria.v12i4a5484.

Santos, F. C. O.; Mendonça, C. L.; Silva Filho, A. P.; Carvalho, C. C. D.; Soares, P. C.; Afonso, J. A. B. 2011. Indicadores bioquímicos e hormonais de casos naturais em toxemia da prenhez em ovelhas. Pesquisa Veterinária Brasileira, 31: 974-980. Doi: 10.1590/S0100736X2011001100006.

Santos, R. P.; Sousa, L. F.; Sousa, J. T. L.; Andrade, M. E. B.; Macedo Júnior, G. L.; Silva, S. P. 2015. Parâmetros sanguíneos de cordeiros em crescimento filhos de ovelhas suplementadas com níveis crescentes de propilenoglicol. Revista Brasileira de Ciências Agrárias, 10: 473-478. Doi: 10.5039/agraria.v10i3a4924.

Silva, J. S. C.; Guaraná, E. L. S.; Lemos, V. F.; Soares, P. C.; Afonso, J. A. B.; Mendonça, C. L. 2013. Metabolismo energético, proteico e mineral de ovelhas Santa Inês hígidas e com mastite subclínica. Pesquisa Veterinária Brasileira, 33, 1087-1096. Doi: 10.1590/S0100736X2013000900007.

Silva, N. C. D.; Harter, C. J.; Resende, K. T. 2018. Utilização de parâmetros bioquímicos na avaliação do status metabólico em animais com restrição nutricional. Ciência Animal, 28: 114-129. Disponível em: http://www.uece.br/cienciaanimal.

Taghipour, B.; Seifi, A. H.; Mohri, M.; Farzaneh, N.; Naserian, A. 2010.Variations of energy related biochemical metabolites during periparturition period in fat-tailed baloochi breed sheep. Iranian Journal of Veterinary Science and Technology, 2: 85-92. Doi: 10.22067/ veterinary.v2i2.8368.

Varanis, L. F. M. 2018. Prospecção de metabólitos sanguíneos referenciais para ovinos em distintas categorias. Uberlândia: Universidade Federal de Uberlândia, 90f. Dissertação Mestrado. Disponível em: https:// repositorio.ufu.br/handle/123456789/21703. 